\title{
Valeur diagnostique actuelle de la réaction de fixation du complément dans le kyste hydatique
}

\author{
par J. COUDERT, J. DESPEIGNES et R. BATTESTI \\ (Laboratoire de Parasitologie, Faculté de Médecine de Lyon, F 69 -Lyon).
}

\begin{abstract}
Résumé
Ce travail repose sur l'analyse des résultats de la réaction de fixation du complément pratiquée sur le sérum de 135 malades atteints d'affections variées et de 63 malades opérés dont les kystes hydatiques ont été examinés du point de vue anatomique.

La réaction de fixation du complément s'est toujours avérés positive quand il s'agissait de kystes vivants ayant une membrane germinative intacte; les taux d'anticorps les plus élevés ont été obtenus chez les malades dont les kystes étaient rompus ou fistulisés dans un organe de voisinage.

Des réactions sérologiques répétées dans les mois ou les années qui ont suivi l'intervention chirurgicale ont montré que la guérison s'accompagnait d'une baisse puis d'une disparition des anticorps et que les récidives étaient annoncées par une remontée des anticorps.

Mais dans un certain nombre de cas, la réaction de fixation du complément s'est avérée négative: il s'agissait de malades opérés de kystes morts, nécrosés et également de malades porteurs de calcifications intrahépatiques découvertes fortuitement.
\end{abstract}

\section{Summary}

This work lies upon the analysis of the fixation reaction of the complement practised on the serums of 135 patients suffering from various diseases and of 63 operated patients whose hydatic cysts have been examined from an anatomic point of view. 
The fixation reaction of the complement always revealed positive when living cysts having a sound germinative membrane were concerned; the highest rates of antibodies have been obtained among patients whose cysts were broken or burst in a neighbouring organ.

Serologic reactions repeated in the months of years which followed the intervention have shown that the return to health was accompanied by a diminution, then the disappearance of the antibodies and the repetitions of illness were annonced by an increase of the antibodies.

But in a certain number of cases the fixation reaction of the complement has turned out to negative. It concerned patients operated from dead necrosis cysts and also patients bearing hepatic calcifications discovered by chance.

Les premiers essais de diagnostic sérologique du kyste hydatique remontent à de nombreuses années puisque Weinberg en 1909 avait imaginé une réaction de fixation du complément avec le sérum des malades en utilisant comme antigène du liquide hydatique frais. Mais ces dernières années nous avons considérablement perfectionné les techniques de fixation du complément pour le diagnostic des maladies parasitaires: la réaction que nous employons pour le diagnostic de la maladie hydatique est beaucoup plus sensible et fidèle que l'ancienne réaction de Weinberg. Nous utilisons comme antigène un extrait total de kyste hydatique humain après avoir vérifié l'intégrité du parasite, membranes et scolex en particulier. Cet antigène est titré ; la constance de sa valeur antigénique nous permet d'avoir des résultats comparables chez le même malade.

Dans ce travail qui porte sur 200 réactions sérologiques pratiquées ces trois dernières années sur le sérum de malades provenant des différents services des hôpitaux de Lyon, nous nous proposons de confronter les résultats sérologiques avec les données anatomiques et cliniques relatives à chaque cas.

Pour 135 malades atteints d'affections variées et parfois suspects d'hydatidose, sans toutefois qu'une intervention chirurgicale ait pu en faire la preuve, nous avons obtenu une interprétation valable de nos résultats sérologiques en fonction du seul contexte clinique.

Mais pour 63 malades atteints de kystes hydatiques opérés nous avons pu faire l'examen anatomique de la pièce opératoire et nous avons abouti à des conclusions formelles sur la valeur de la réaction de fixation du complément. Pour chacun de ces malades nous avons pratiqué un dosage des anticorps avant l'intervention et, pour certains d'entre eux, deux ou plusieurs examens sérologiques dans les mois ou les années qui suivirent.

C'est donc essentiellement sur ces 63 observations de kystes hydatiques vérifiés anatomiquement que repose notre travail de confrontation anatomo-clinique et sérologique. 


\section{Kystes hydatiques vérifiés par l'intervention : 63 observations}

\section{Résultats d'ensemble.}

Les 63 cas de kystes hydatiques vérifiés anatomiquement se répartissent ainsi suivant leur localisation:

39 kystes hydatiques du foie

6 kystes hydatiques péritonéaux

4 kystes hydatiques du poumon

4 kystes hydatiques de la rate

2 kystes hydatiques du rein

4 kystes hydatiques osseux

4 kystes hydatiques de localisations diverses $=$ thymus, corps thyroïde, région ombilicale, creux poplité.

La réaction de fixation du complément a été positive 57 fois, soit dans $90 \%$ des cas avec des taux d'anticorps compris entre $1 / 4$ et $1 / 256$.

Mais sept fois la réaction de fixation du complément est restée négative : il s'agissait de malades porteurs de kystes hydatiques anciens, nécrosés.

Les taux d'anticorps obtenus ne semblent pas en rapport avec la localisation ou le volume des kystes, mais plutôt avec leur état anatomique. En effet, 54 fois, soit dans $85 \%$ des cas, nous avons pu faire une étude anatomique des kystes opérés qui se présentent essentiellement sous deux aspects différents: les kystes vivants et les kystes morts.

Les kystes vivants ont une paroi mince à la face interne de laquelle on peut reconnaître la membrane germinative qui, dans le cas du kyste fertile, bourgeonne des scolex. Ils contiennent un liquide eau de roche, très antigénique, et souvent des vésicules filles. La fissuration de ces kystes stimule la production des anticorps; leur rupture est à l'origine de chocs anaphylactiques ou d'échinococcose secondaire.

Les kystes morts, au contraire, possèdent une paroi épaisse, scléreuse, parfois calcifiée ; leur contenu est épais, visqueux et fait de débris nécrosés où l'on ne reconnaît plus ni membrane germinative, ni scolex. Le diagnostic de kyste hydatique est assuré seulement par l'aspect stratifié de la membrane anhiste et parfois par la présence de crochets. Ces kystes ne possèdent plus de potentiel évolutif.

A l'état anatomique de ces kystes correspondent des résultats sérologiques particuliers : en effet, les taux d'anticorps obtenus dans le cas de kystes morts sont nettement plus bas que dans le cas de kystes vivants et, parmi ces derniers, ce sont les kystes compliqués, fistulisés dans un organe de voisinage qui donnent les taux d'anticorps les plus élevés.

Cette correspondance assez étroite entre le taux d'anticorps des sérums et l'évolution anatomo-clinique nous a permis de classer nos 63 observations de kystes hydatiques en trois groupes principaux :

Les kystes vivants fissurés ou fistulisés dans un organe voisin. 
Les kystes hydatiques vivants bien circonscrits.

Les kystes hydatiques morts.

\section{Les kystes hydatiques vivants, fistulisés dans un organe de voisinage :}

Les observations sont au nombre de 21 ainsi réparties:

2 kystes hydatiques du foie fistulisés dans les bronches;

2 kystes hydatiques à localisation double, hépatique et pulmonaire ;

4 kystes hydatiques du foie rompus dans les voies biliaires;

4 kystes hydatiques du foie rompus dans le péritoine;

7 échinococcoses péritonéales secondaires à un kyste hydatique du foie opéré dont 2 à localisations multiples ;

1 kyste hydatique du rein rompu dans les voies biliaires;

1 kyste hydatique du fémur ayant envahi les parties molles.

Dans tous ces cas une solution de continuité au niveau des membranes s'est produite à un certain moment de l'évolution du kyste, donnant issue au liquide hydatique et parfois à des éléments fertiles. Ceci explique l'élévation importante des anticorps dont les taux moyens se situaient entre le $1 / 64$ et le $1 / 256$. C'est chez ces malades qu'ont été observés des phénomènes allergiques ou même des chocs anaphylactiques; c'est aussi chez eux qu'ont été observées des échinococcoses secondaires.

Voici quelques observations de kystes rompus ou fistulisés dans un organe de voisinage :

Malade originaire d'Afrique du Nord, hospitalisé à la suite d'un choc anaphylactique spontané avec perte de connaissance et urticaire généralisé. Il existe un foie débordant d'un travers de main et une énorme lacune du lobe droit à la scintigraphie. La réaction de fixation du complément est positive à $1 / 64$ et l'intradermo-réaction de Casoni très positive. L'intervention montre un Kyste hydatique de la convexité du foie fistulisé dans les bronches. Le malade meurt de choc anaphylactique au cours de l'exérèse.

Malade hospitalisé pour douleurs de l'hypochondre droit et dyspnée d'effort. L'examen montre un très gros foie et la scintigraphie une énorme lacune du lobe droit. Une réaction de fixation du complément positive à $1 / 128$ confirme le diagnostic d'Hydatidose. L'intervention révèle un Kyste hydatique du foie occupant tout le lobe droit et rempli d'une centaine de vésicules filles. L'exploration de la cavité montre une large fistule biliaire.

Une échinococcose secondaire peut se manifester dans les mois ou les années qui suivent la rupture du kyste initial, entraînant parfois plusieurs localisations:

Malade d'origine tunisienne, opérée d'un Kyste hydatique du foie deux ans auparavani et hospitalisée pour une tumeur abdominale. La réaction de fixation du complément positive à 1/64 est nettement en faveur d'une récidive. L'intervention montre la présence d'un énorme Kyste hydatique péritonéal rempli de vésicules. 
Jeune femme algérienne hospitalisée à la suite d'une hémiplégie avec perte de connaissance. A l'examen, il existe une tumeur pelvienne et rétrocrurale dont la ponction retire un liquide «eau-de-roche ». Pendant son séjour à l'Hôpital la malade évacue par le vagin une vésicule dont le contenu renferme des scolex. La réaction de fixation du complément est positive à $1 / 256$. Neuf mois après une première intervention, la malade élimine à nouveau par voie urinaire des membranes dont l'examen confirme la nature hydatique. La réaction de fixation du complément est toujours positive à $1 / 128$ et traduit la gravité de la maladie hydatique à localisations pelviennes multiples et probablement aussi cérébrales.

Dans tous ces cas de kystes hydatiques vivants, fissurés ou fistulisés dans un organe voisin, le diagnostic d'hydatidose a pu être confirmé avant l'intervention par la réaction de fixation du complément qui a révélé chaque fois un taux d'anticorps élevé.

\section{Les kystes hydatiques vivants bien circonscrits :}

Les observations sont ici au nombre de 27 ainsi réparties:

18 kystes hydatiques du foie;

2 kystes hydatiques du poumon;

3 kystes hydatiques osseux (fémur, aile iliaque et $12^{\circ}$ vertèbre dorsale) ;

1 kyste hydatique du corps thyroïde;

1 kyste hydatique du rein;

1 kyste hydatique du thymus.

Chez ces malades les taux d'anticorps s'échelonnaient entre le 1/8 et le 1/32. Ces taux relativement peu élevés s'expliquent par l'isolement relatif du parasite dans l'organisme. Ces kystes uniques, parfois multiples, étaient souvent entourés d'un périkyste conjonctif épais, peu propice à la dissémination du parasite : leur contenu révélait cependant la présence de membranes fertiles et de scolex bien vivants.

Peu de signes cliniques, en général, attiraient l'attention comme en témoignent ces quelques observations :

Berger des Basses-Alpes chez qui un examen radiologique systématique découvre une image ronde du poumon. La réaction de fixation du complément est positive à $1 / 8$. L'intervention permet l'exérèse d'un Kyste hydatique de la base à paroi épaisse, sans communication bronchique.

Nord-Africain hospitalisé pour douleurs sus trochantériennes. La radiographie montre la présence d'un Kyste de l'aile iliaque sans envahissement articulaire. Le taux d'anticorps en réaction de fixation du complément est à $1 / 8$ et l'intradermo-réaction de Casoni positive. L'intervention chirurgicale permet l'exérèse totale du kyste et six mois plus tard les réactions sérologiques sont négatives.

L'exérèse de ces kystes bien limités fut, en général, simple et on ne signala pas de récidives ultérieures. Les anticorps trouvés à des taux relativement peu élevés avant l'intervention disparurent progressivement dans les mois qui suivirent. 


\section{Les kystes hydatiques morts :}

Ces kystes possèdent un périkyste scléreux, souvent calcifié et un contenu épais, de consistance et de couleur « mastic ». On ne reconnaît plus les éléments cellulaires de la membrane germinative et la membrane anhiste elle-même est altérée.

La plupart de ces kystes involués sont de siège hépatique ou splénique; leur découverte a été le plus souvent fortuite, à l'occasion d'un examen radiologique, sans qu'aucun signe clinique d'appel n'ait signalé leur présence.

Nous avons relevé quinze observations de kystes morts, ainsi réparties :

10 kystes hydatiques du foie;

4 kystes hydatiques de la rate;

1 kyste hydatique du creux poplité.

La réaction de fixation du complément n'a été positive que huit fois avec des taux d'anticorps bas, allant de $1 / 4$ à $1 / 8$ :

Algérien hospitalisé pour splénomégalie isolée avec à la radiographie une image arrondie aux contours calcifiés. La réaction de fixation du complément est positive à $1 / 8$ et l'intradermo-réaction de Casoni positive. L'intervention découvre un Kyste hydatique de la rate à parois calcifiées et à contenu nécrotique sans éléments identifiables.

Sept fois, soit dans la moitié des cas de kystes morts, les réactions sérologiques demeurèrent négatives avant l'intervention. Chez quatre de ces malades l'intradermoréaction de Casoni, par contre, avait entraîné une réaction immédiate, deux fois fortement positive et deux fois de façon plus discrète. Ce fait serait en faveur de la persistance de l'hypersensibilité cutanée alors que les anticorps circulants ont disparu.

Chez trois malades de nouvelles réactions sérologiques pratiquées après l'intervention se révélèrent positives pour la première fois à des taux très bas ne dépassant pas $1 / 4$ :

Algérienne hospitalisée pour une image calcifiée sous-diaphragmatique découverte à l'occasion d'un examen radiologique systématique. La cartographie montre une lacune intrahépatique. La réaction de fixation du complément est négative mais l'intradermo-réaction de Casoni est positive. L'intervention permet l'exérèse d'un Kyste hydatique du volume d'une orange à parois calcifiées et à contenu nécrotique. Quelques jours après l'intervention une nouvelle réaction de fixation du complément s'avère positive à $1 / 4$.

C'est donc bien à ces kystes morts qu'il faut rapporter les $10 \%$ de réactions sérologiques négatives parmi les 63 observations de kystes hydatiques opérés. En effet, il est de règle de constater la disparition des anticorps circulants dans l'année qui suit l'exérèse ; en cas de mort du parasite cette disparition a lieu également dans 
des délais difficiles à préciser, mais qui expliquent que les kystes morts depuis plusieurs années restent muets du point de vue sérologique.

\section{Valeur pronostique actuelle de la réaction de fixation du complément :}

Si les réactions sérologiques permettent le plus souvent de confirmer le diagnostic de kyste hydatique, elles permettent aussi après l'acte chirurgical de surveiller l'évolution de la maladie et de prévoir les récidives.

Chez 17 malades nous avons pu confronter la courbe des anticorps et l'évolution clinique de la maladie pendant une période allant de quelques mois à plusieurs années.

Au cours de la période post-opératoire immédiate nous avons observé quatorze fois une élévation nette des anticorps dont le taux a doublé ou quadruplé :

- Chez un jeune Algérien porteur d'un kyste hydatique du rein avec hydronéphrose la réaction de fixation du complément était positive à $1 / 128$; l'intervention découvrit un volumineux kyste bourré de vésicules. Huit jours après l'exérèse le taux des anticorps s'était élevé à $1 / 256$.

- Chez un malade atteint de kyste hydatique pulmonaire la réaction de fixation du complément passa de $1 / 8$ avant l'intervention à $1 / 32$ les jours suivants.

- Chez trois malades porteurs de kystes nécrosés dont les réactions séro'ogiques étaient négatives avant l'intervention, le taux des anticorps monta à $1 / 4$ dix jours après l'exérèse.

Cette augmentation des anticorps après l'acte opératoire est transitoire et n'annonce pas une récidive. Elle est provoquée par la libération d'une faible quantité d'antigène inévitable au cours des manipulations opératoires.

L'évolution secondaire s'est faite chez huit malades vers la baisse régulière des anticorps trouvés à des taux très faib'es, $1 / 4$ ou $1 / 2$, plusieurs mois après l'intervention, mais la réaction de fixation du complément ne devint négative qu'environ un an après l'exérèse du kyste. Ces malades ne présentèrent aucune récidive ultérieure et la guérison clinique confirma les données bio'ogiques:

- Ce fut le cas de deux Algériens atteints d'échinococcose osseuse dont le taux d'anticorps baissa en quelques mois, de trois ma'ades opérés plusieurs années auparavant d'hydatidose hépatique dont les réactions sérologiques étaient devenues négatives. La guérison a pu survenir rapidement après l'intervention malgré la gravité de l'hydatidose : chez cet Algérien atteint d'hydatidose péritonéa'e secondaire à un kyste hydatique de la rate la réaction de fixation du complément est positive à 1/32. L'intervention ramène plusieurs kystes péritonéaux. Un mois plus tard, le taux des anticorps n'est p'us qu'à $1 / 8$ et les réactions sérologiques sont négatives trois ans plus tard, alors qu'une laparotomie exploratrice ne montre la persistance que d'un petit kyste calcifié. 
Mais chez neuf malades la courbe des anticorps, après avoir amorcé une décroissance momentanée, est remontée six mois à un an après l'exérèse du kyste initial :

Ainsi, chez deux malades atteints d'échinococcose osseuse, il fallut trois interventions pour éliminer le parasite et les récidives furent chaque fois annoncées par l'augmentation du taux des anticorps. Sept autres malades qui avaient été opérés d'un kyste hydatique du foie développèrent secondairement une hydatidose péritonéale. Le taux d'anticorps s'éleva de $1 / 8$ à $1 / 64$ assez rapidement, précédant les signes cliniques évocateurs de cette nouvelle localisation.

Chez un malade opéré d'un kyste hydatique du foie on découvrit cinq ans plus tard une tumeur de la fosse iliaque, la réaction de fixation du complément était positive de 1/32. L'intervention ramena une multitude de kystes péritonéaux. Mais huit mois plus tard le taux d'anticorps est stationnaire et une nouvelle intervention permet encore d'enlever plusieurs kystes péritonéaux, la plupart fertiles.

Ainsi, la persistance d'un taux élevé d'anticorps en réaction de fixation du complément est le témoin de l'évolutivité de l'hydatidose et doit faire redouter une récidive.

\section{Réactions sérologiques systématiques : 135 observations}

Mais à côté de ces 63 observations de kystes hydatiques confirmés anatomiquement notre enquête a porté sur 135 réactions de fixation du complément pratiquées chez des malades pour lesquels fut évoqué le diagnostic d'hydatidose.

- Parmi ces 135 examens 95 furent négatifs. Mais dans 30 cas seulement, une intervention chirurgicale ou une laparotomie exploratrice furent pratiquées et vérifièrent l'absence de kyste hydatique.

Il s'agissait en particulier de : quatorze cancers du foie, primitifs ou secondaires, de trois cirrhoses, de deux kystes solitaires du foie, de deux abcès amibiens, de deux tumeurs bénignes du poumon, d'un cancer péritonéal, d'un pseudo-kyste du pancréas, d'une tumeur cérébrale, de trois malformations des voies biliaires, d'un phéochromocytome.

Chez quatre seulement, parmi ces 95 malades, fut évoqué le diagnostic d'hydatidose: il s'agissait d'Algériens qui présentaient une image calcifiée intrahépatique découverte fortuitement. Mais, en raison de l'absence de manifestations cliniques, ces malades ne furent pas opérés. Ces quatre observations nous montrent encore que certains malades, porteurs de kystes hydatiques anciens, calcifiés, ne présentent plus d'anticorps dans leur sérum et qu'ils paraissent guéris de leur maladie hydatique.

- 40 fois la réaction cie fixation du complément s'avère positive, 28 fois à des taux faibles de $1 / 2$ à $1 / 4$ et 12 fois à des taux supérieurs.

Les malades qui présentaient des taux d'anticorps de $1 / 8$ et davantage étaient vraisemblablement porteurs de kystes hydatiques, étant donné l'origine algérienne de la plupart et les signes cliniques évocateurs; mais le diagnostic ne put être vérifié par 
l'intervention et les explorations radiologiques demeurèrent insuffisantes comme en témoignent ces quelques observations :

Musulman présentant une image arrondie de la base pulmonaire droite dont la ponction retira un liquide purulant. La réaction de fixation du complément était positive à 1/16. Le malade refusa l'intervention.

Jeune Algérien opéré pour une hernie inguinale chez lequel une réaction sérologique systématique montra un taux d'anticorps élevé, 1/128. Une scintigraphie révéla, en plus de petites lacunes intrahépatiques, une image splénique très suspecte. Mais la malade quitta le Service et les investigations en restèrent là.

Espagnol porteur d'un ulcère duodénal en même temps que d'une déformation de la coupole diaphragmatique droite. Une réaction de fixation du complément est trouvée positive à $1 / 8$. La cartographie révéla une lacune de la partie supérieure du foie très évocatrice d'un kyste du dôme hépatique.

Algérien chez qui on découvre, à l'occasion d'un lumbago, une image ronde calcifiée de l'hypochondre droit. Une scintigraphie montre une lacune dans la partie postérieure du lobe hépatique droit. La réaction de fixation du complément est positive à $1 / 4$ et l'intradermo-réaction de Casoni très positive. Mais la latence clinique de cette Hydatidose probable en fit différer l'intervention.

Enfin, 28 fois la réaction de fixation du complément se révéla positive à $1 / 2$ ou $1 / 4$.

Parmi ces malades onze étaient atteints d'affections diverses : typhoïde, paludisme, infections urinaires, six étaient porteurs de tumeurs malignes et six d'une parasitose : bilharziose intestinale, anguillulose, distomatose, taeniasis...

Enfin, cinq malades présentaient des images calcifiées de la région hépatique.

Pour ces 28 malades le diagnostic de kyste hydatique ne fut pas évoqué ; pourtant les circonstances étiologiques rendaient possible la coexistence d'une affection aiguë et d'un kyste hydatique latent.

Tous ces malades, en effet, étaient originaires d'Algérie, région de forte endémie hydatique, et l'on sait que ies manifestations cliniques de l'hydatidose sont relativement rares par rapport aux kystes latents découverts fortuitement. C'est sur ces derniers notamment que les réactions sérologiques systématiques peuvent attirer l'attention, permettant ainsi une étude épidémiologique de la maladie.

\section{Conclusion}

L'analyse de nos observations montre que la réaction de fixation du complément s'est avérée positive chez tous les malades atteints de kystes hydatiques vivants avec des taux d'anticorps particulièrement élevés pour les kystes fistulisés dans un organe de voisinage.

La surveillance du taux d'anticorps dans les mois qui suivent l'intervention chirurgicale permet d'affirmer la guérison de la maladie hydatique ou de prévoir les récidives avant l'apparition des manifestations cliniques. 
Mais dans un certain nombre de cas, la réaction de fixation du complément s'est montrée négative, notamment chez les malades opérés de kystes morts ou présentant des calcifications intrahépatiques découvertes fortuitement.

\section{Bibliographie.}

Bettinotti (C. M.) et Bodereau (E. F.), 1957. - Hydatidose. Etude comparative des réactions diagnostiques de laboratoire. Pressa Med. argent. 44, $\mathrm{n}^{\circ}$ 2: 93-97.

Bensted (H. J.), AtKinson (J. D.), 1958. - Le test de fixation du complément pour l'Hydatidose. Action des protéines de l'hôte intermédiaire sur la spécificité de l'antigène du liquide hydatique de mouton. Brit. Méd. J. $\mathrm{n}^{\circ}$ 5090: 203-205.

Biguet (J.), Rose (G.), Capron (A.) et Tran Van Ky (P.), 1965. - Contribution de l'analyse immunoélectrophorétique à la connaissance des antigènes verminaux. Incidences pratiques sur leur standardisation, leur purification et le diagnostic des helminthiases par immunoélectrophorèse. Revue d'Immunologie Paris, 29, 5.

CApron (A.), Verne (A.) et Biguet (J.), 1966. - Le diagnostic immunoélectrophorétique de l'Hydatidose. Le kyste hydatique du foie. S.I.M.E.P. Ed. Lyon.

Chordi (A.) et Gonzales-Castro (J.), 1961. - Le test de fixation du complément dans l'Echinococcose ovine. Rev. Med. Est. G. Navarra, 5: 1-19.

COUderT (J.), 1955. - Antigènes lyophilisés en Parasitologie. La Presse Médicale, 52 : 1080-1081.

COUDERT (J.), 1961. - Utilisation des antigènes lyophilisés pour le diagnostic des maladies parasitaires. Ann. Biol. Clin. 12: 1-9.

Coudert (J.), Despeignes (J.), Ambroise-Thomas (P.) et Battesti (M. R.), 1965. - Valeurs comparées des différentes méthodes de diagnostic immunologique de l'Echinonoccose alvéolaire. Lyon Médical, 52 : 1581-1584.

Garabedian (G. A.), Matossian (R. M.) et Suidan (F. G.), 1959. - A correlative study of immunological tests for the diagnosis of hydatid disease. American J. Trop. Med. Hyg., 8, 67.

KAGAN (I. G.), 1963. - Seminar on immunity to parasitic helminths. VI. Hydatic disease. Exper. Parasitol., 13, 57.

Kagan (I. G.), Norman (L.) et Allain (D. S.), 1960. - Studies on Echinococcosis : serology of crude and fractionated antigens prepared from E. granulosus et E. multilocularis. Amer. J. Trop. Med. Hyg., 9, 248.

Lupascu (G. H.) et PanattesCu (D.), 1968. - Hidatidoza. Editura academiei republicii socialiste romania.

Patel (J.-Cl.), 1961. - Diagnostic biologique de l'Hydatidose. Presse Médicale, 69, $\mathrm{n}^{\circ} 15$ : 688.

PAuluzzI (S.), 1964. - La fissazione del complemento par l'Idatidosi. $\mathrm{I}^{\circ}$ Un micrometodoso semiquantitativo. Boll. Instit. Sieroter. Milan. 43: 1.

Pauluzzi (S.), 1964. - La fissazione del complemento per 1'Idatidosi. II ${ }^{\circ}$. Standardizzazione biologica del potere antigene del liquido cistico e di altri materiali idatidei. Boll. Instit. Sieroter. Milan. 43: 11 . 
Pauluzzi (S.), Sorice (F.), Castagnari il.), Serra (P.), - La fissazione de complemento per l'Idatidosi. IV $^{\circ}$. Risultati diagnostici nella idatidosi umana ottenuti con antigeni idatidei standardi ati non purificati, di varia provenienza liquido cistico, scolici, membrana cuticolare). Annali Sclavo. 8: 74 .

Pautrizel (R.), Bailenger (J.), 1960. - Le diagnostic sérologique de l'Echinococcose par la réaction de fixation du complément technique d'hémolyse et de conglutination. Arch. Intern. H.I.D. 19: 305.

Pautrizel (R.), Balenger (J.), 1961. - Diagnostic immunologique de l'Echinococcose. Ann. Biol. Clin. 19: 243-252.

SAvel (J.), 1961. - Les modalités d'élaboration et de manifestations objectives des anticorps parasitaires. Ann. Biol. Clin., 19: 225-231.

Sorice (F.), Pauluzzi (S.), Castagnari (L.), Tolu (A.), 1965. - La fissazione del complemento per l'Idatidosi. $\mathrm{VI}^{\circ}$. Confronto con la reazion di emoagglutinazione indiretta nella idatidosi humana. Boll. Instit. Sieroter. Milan. 44: 22. 


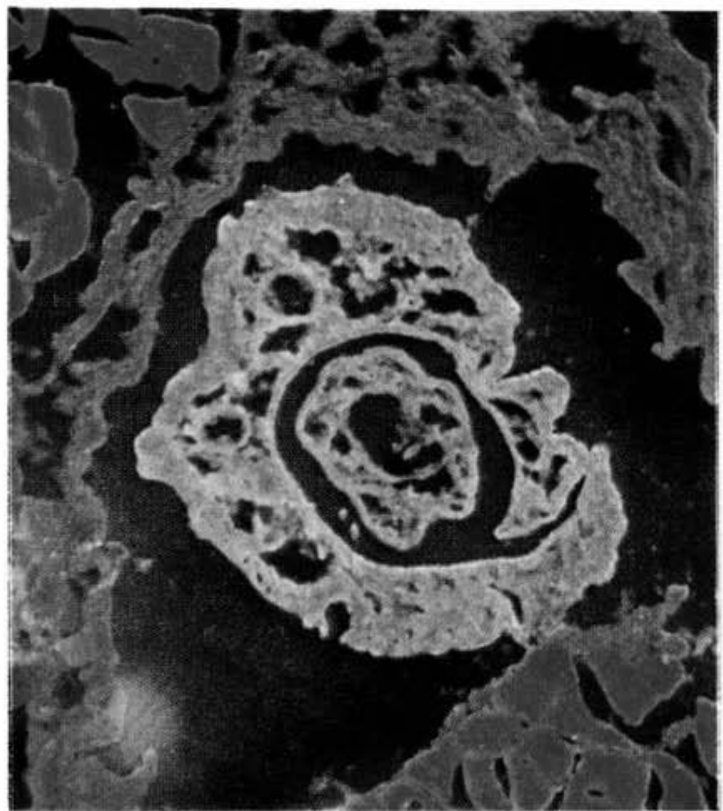

Рното I. - Séro-diagnostic de la bilharziose par immuno-fluorescence sur coupes de Schistosoma haematobium adultes. Réaction positive.

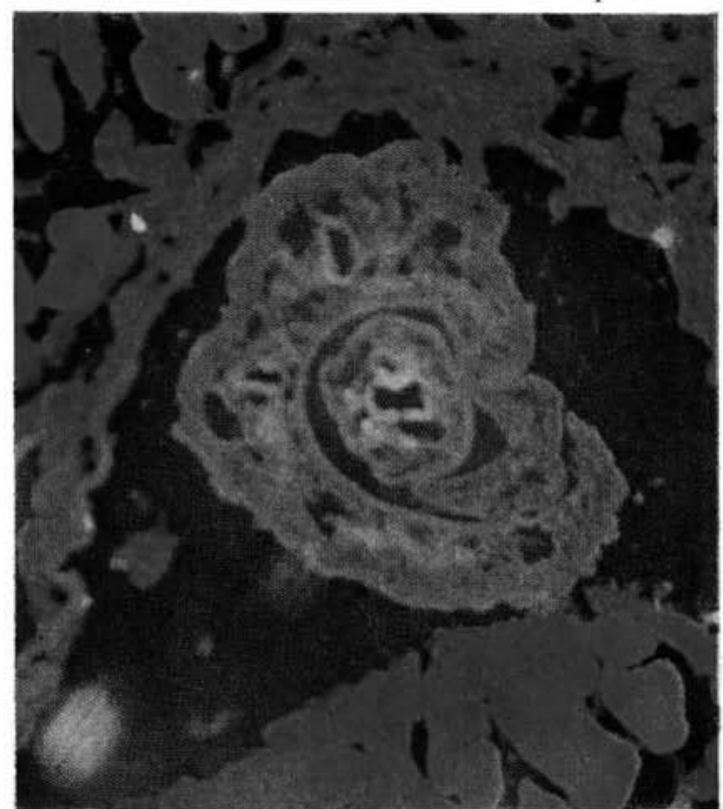

Pнoto II. - Séro-diagnostic de la bilharziose par immuno-fluorescence sur coupes de Schistosoma haematobium adultes. Réaction négative. 